\title{
Clinical Significance of Serum Interleukin-31 and Interleukin-33 Levels in Patients of Endometrial Cancer: A Case Control Study
}

\author{
Xi Zeng, ${ }^{1}$ Zhu Zhang, ${ }^{1}$ Qian-Qian Gao, ${ }^{1}$ Yan-Yun Wang, ${ }^{2}$ Xiu-Zhang Yu, ${ }^{1}$ \\ Bin Zhou, ${ }^{2}$ and Ming-Rong $\mathrm{Xi}^{1}$ \\ ${ }^{1}$ Department of Gynecology and Obstetrics, The West China Second University Hospital, Sichuan University, Key Laboratory of \\ Birth Defects and Related Diseases of Women and Children (Sichuan University), Ministry of Education, Chengdu 610041, China \\ ${ }^{2}$ Department of Molecular Translational Medicine, The West China Institute of Women and Children's Health, \\ West China Second University Hospital, Sichuan University, Chengdu, China
}

Correspondence should be addressed to Ming-Rong Xi; jzzqmr@126.com

Received 26 October 2015; Revised 4 April 2016; Accepted 13 April 2016

Academic Editor: Lance A. Liotta

Copyright (C) 2016 Xi Zeng et al. This is an open access article distributed under the Creative Commons Attribution License, which permits unrestricted use, distribution, and reproduction in any medium, provided the original work is properly cited.

\begin{abstract}
Aims. Previous evidence has proved that interleukin-31 (IL-31) and interleukin-33 (IL-33) can be potential markers in some cancers' formulation. We aimed to determine the potential role of IL-31 and IL-33 in prognosis of endometrial cancer patients. Methods. Serum samples were collected from 160 patients with endometrial cancer and 160 healthy controls. The ELISA kits (Raybio ${ }^{\circledR}$ Systems) specific for human IL-31 and human IL-33 were used. Serum levels of tumor markers (CEA, CA-125, and CA19-9) were measured by chemiluminescence immunoassay. A two-side $P$ value $<0.05$ was indicated to be significant. Results. Serum levels of IL-31 and IL-33 in patients were significantly elevated compared to those of healthy controls. The interleukin levels were also related to clinical characteristics, including tumor stages, depth of invasion, and existence of node metastases and distant metastases. The sensitivity and specificity of IL-31 and IL-33 were higher than the counterparts of tumor markers, both separately and in combination of IL-31, IL-33, and the clinical markers. Conclusions. This report is the first one mentioning the possible association between serum IL-31 and IL-33 and endometrial cancer. With their sensitivity and specificity, the interleukins may be useful biomarkers for endometrial cancer's prognosis.
\end{abstract}

\section{Introduction}

Endometrial cancer (EC) is the second most common female cancer with incidence varying among regions. More than 287,100 new EC cases are diagnosed each year, which are more common in developed countries with the risk of endometrial cancer being $1.6 \%$ and oppositely less common in developing countries with the lowest rates of $0.6 \%$ [1].

EC occurs most frequently during perimenopause and menopause, between the ages of 50 and 65 worldwide [2]. The worldwide median age at diagnosis of endometrial cancer is 63 years of age [3]. Based on a recent report, EC commonly occurs earlier in Chinese women than those in western countries [4].

The mechanism for the formulation of EC is still not well clear. Endometrial inflammation is considered as an important risk factor for EC [5]. Pathologic angiogenesis has been implicated on the development of chronic inflammatory diseases and several cancers. Some cytokines involved mediating between inflammation and angiogenesis, including interleukins $[6,7]$. Previous researches have already revealed neoangiogenesis closely associated with the growth and the development of metastasis of various tumors, including EC $[8,9]$.

The diagnosis of EC is generally according to the clinical manifestation like postmenopausal bleeding or the serum levels of some tumor markers, while about $15 \%$ of ECs occur in women without vaginal bleeding [10]. And the role of tumor markers in endometrial cancer is still in research. Previously, literatures reported the role of different serum markers in endometrial cancer such as carcinoembryonic antigen (CEA), carbohydrate antigen-125 (CA-125), and carbohydrate antigen 19-9 (CA19-9), resulting in elevation in 
only $20 \%$ to $30 \%$ of patients [11]. Thus, some more efforts should be exerted on finding a new marker with better sensitivity and specificity in the early diagnosis of endometrial adenocarcinoma.

According to the relationship between the inflammation, angiogenesis, and EC, we supposed that some cytokines perhaps could be used as new markers.

Interleukin-31 (IL-31) belongs to the IL-6 family cytokines and is mainly secreted by activated $\mathrm{CD} 4^{+} \mathrm{T}$ helper (Th) cells [12]. IL-31 acts through the heterodimeric receptors of IL-31 (IL-31R) and oncostatin receptor (OSMR) expressed on IL-31 activated monocytes and expressed on epithelial cells [13]. IL31 can significantly upgrade the gene and protein expression of vascular endothelial growth factor (VEGF) and epidermal growth factor (EGF) [14].

Interleukin-33 (IL-33) is a new member of the IL-1 family of cytokines [15]. IL-33, passing signal via ST2 receptor, is considered closely associated with IL-18, one of the most novel members in the family [16]. Previous researches illustrated that the elevated serum IL-18 played an important role in a variety of cancers [17-19], and a similar function of IL33 is still under debate. Some following researches detected elevated serum IL-33 in various tissues of lung, stomach, spinal cord, skin, and brain, also in cells like epithelial cells lining bronchus and smooth muscle cells [20]. Küchler et al. implied that the expression of IL-33 is regulated by vascular endothelial cells in vivo [21]. Choi et al. [22] reported that IL33 promotes angiogenesis.

Thus, researches labeled IL-31 and IL-33 with antiproliferative and angiogenic factors. More following evidences have proved that IL-31 and IL-33 play an important role in some cancers' formulation, like lung cancer, gastric cancer, and squamous cancer [23-25]. However, there is no research on the relationship of IL-31 and IL-33 with EC.

In this study, the serum levels of IL-31 and IL-33 in EC patients were studied and assessed the interrelationship with clinical significance and some tumor markers. We also measured levels of these cytokines in healthy persons. This study provides new insight into the mechanism of function of new angiogenic cytokines in EC. Based on English literature reviews, this is the first research reported on the association between IL-31 and IL-33 and EC.

\section{Materials and Methods}

2.1. Patients and Blood Samples. After acquiring the consent from ethics committee of the West China Second University Hospital, Sichuan University, China (number 2013-036), we selected 160 patients of EC aged between 18 and 80 years who were admitted to our hospital from Jan. 2014 to Jan. 2015. Those patients had been diagnosed by curettage of uterus and were double checked by two pathologic doctors. The stage of the tumor was classified based on the International Federation of Gynecology and Obstetrics (FIGO) criteria for adenocarcinoma of the endometrium (FIGO, 2009) [26]. There was no radiation or chemotherapy before.

Another 160 sex and age matched females, seeking for physical examination at our hospital, were recruited as healthy controls, who had no history of adenocarcinomas or gynecological diseases. The results of gynecologic examinations and transvaginal sonographies in the controls were normal.

All the subjects consented to join this test which was done in agreement with the Declaration of Helsinki. With obtaining written informed consent, a $7 \mathrm{~mL}$ blood sample was collected from the antecubital vein and stored at $-80^{\circ} \mathrm{C}$ until testing. Throughout the research, the patients' anonymity was preserved.

2.2. Detection of Interleukins and Tumor Markers (CEA, CA125, and CA19-9). The ELISA kits (Raybio Systems) specific for human IL-31 and human IL-33 were used to detect serum IL-31 and IL-33 levels, respectively. The sensitivity limits of the ELISA kits were separately from $4.92 \mathrm{pg} / \mathrm{mL}$ to $1200 \mathrm{pg} / \mathrm{mL}$ (IL-31) and from $2.05 \mathrm{pg} / \mathrm{mL}$ to $500 \mathrm{pg} / \mathrm{mL}$ (IL33). The intra-assay and interassay reproducibilities of the ELISA kits were $9 \%$ and $11 \%$ (IL-31) and $8 \%$ and $11 \%$ (IL-33). The levels of tumor markers (CEA, CA-125, and CA19-9) were measured by chemiluminescence immunoassay. Our clinical laboratory has been certificated with ISO15189. The reagents manufactured by SIEMENS (Massachusetts, USA) were used in the test of the tumor markers. The sensitivity limit of the CEA reagent was from $0.5 \mathrm{ng} / \mathrm{mL}$ to $100 \mathrm{ng} / \mathrm{mL}$ (interassay reproducibility $3.3 \%$, intra-assay reproducibility $3.8 \%$ ). The CA19-9 reagent's sensitivity limit was from $1.2 \mathrm{U} / \mathrm{mL}$ to $700 \mathrm{U} / \mathrm{mL}$ (interassay reproducibility $8 \%$, intra-assay reproducibility $4 \%$ ). The sensitivity limit of the CA-125 reagent was from $2 \mathrm{U} / \mathrm{mL}$ to $600 \mathrm{U} / \mathrm{mL}$ (interassay reproducibility $4 \%$, intra-assay reproducibility $3 \%$ ). The normal standards were set as follows: CEA $<2.5 \mathrm{ng} / \mathrm{mL}, \mathrm{CA}-125<35 \mathrm{U} / \mathrm{mL}$, and CA19-9 $<30 \mathrm{U} / \mathrm{mL}$. All tests were performed based on the instruction.

2.3. Statistical Analysis. The SPSS software (Version 22.0, IBM Company, USA) was used for data analysis. Data is shown as mean \pm standard deviation (range). The categorical variables between the patients and controls were performed with chi-squared tests. After a normality test of the original data, the correlation between the clinical factors and the levels of IL-31 and IL-33 was analyzed by independent-samples $t$ test. The correlation between the tumor markers and the interleukin levels was analyzed with Student's $t$-test. A cutoff value was determined by analysis of receiver operating characteristic (ROC). Logistic analysis was adopted in the multitarget diagnostic value evaluation. A two-side $P$ value $<$ 0.05 was indicated to be significant. The figures were made by the GraphPad Prism 5 software package (GraphPad Software Inc., USA).

\section{Results}

3.1. Serum IL-31 and IL-33 in Patients and Controls. The mean age of diagnosis in EC was 54.13 years (range from 37 to 74 years). To be specific, there were 44 patients with tumor stage I; 44 patients had stage II disease; 32 patients were with stage III; and 40 patients were with stage IV. The healthy controls were with a median age of 54.03 years (range from 38 to 74 years) (Table 1). 
TABLE 1: Description characters of the patients and controls. Data is shown as mean \pm standard deviation (SD).

\begin{tabular}{|c|c|c|c|}
\hline Characters & Number of patients (\%) & Number of controls (\%) & $P$ value \\
\hline Sample size & 160 & 160 & \\
\hline Mean age $\pm S D$ (range) (years) & $54.13 \pm 7.78(37-74)$ & $54.03 \pm 7.94(38-74)$ & 0.826 \\
\hline $\mathrm{BMI}$ mean $\pm \mathrm{SD}\left(\mathrm{kg} / \mathrm{m}^{2}\right)$ & $24.71 \pm 2.76$ & $24.88 \pm 2.76$ & 0.948 \\
\hline Family history of cancer & & & 0.136 \\
\hline Yes & $24(15 \%)$ & $8(5 \%)$ & \\
\hline No & $136(85 \%)$ & $152(95 \%)$ & \\
\hline Menopausal status & & & 0.651 \\
\hline Premenopausal & $88(55 \%)$ & $96(60 \%)$ & \\
\hline Postmenopausal & $72(45 \%)$ & $64(40 \%)$ & \\
\hline History of pregnancy & & & 0.556 \\
\hline Yes & $152(95 \%)$ & $148(92.5 \%)$ & \\
\hline No & $8(5 \%)$ & $12(7.5 \%)$ & \\
\hline \multicolumn{4}{|l|}{ Uterine bleeding } \\
\hline Yes & $120(75 \%)$ & & \\
\hline No & $40(25 \%)$ & & \\
\hline \multicolumn{4}{|l|}{ FIGO stage } \\
\hline I & $44(27.5 \%)$ & & \\
\hline II & $44(27.5 \%)$ & & \\
\hline III & $32(20 \%)$ & & \\
\hline IV & $40(25 \%)$ & & \\
\hline \multicolumn{4}{|l|}{ Histology } \\
\hline Adenocarcinoma & $160(100 \%)$ & & \\
\hline Nonadenocarcinoma & $0(0 \%)$ & & \\
\hline
\end{tabular}

TABLE 2: Description of the serum levels of IL-31 and IL-33 in patients and controls (pg/mL). Data is shown as mean \pm standard deviation (range).

\begin{tabular}{lcccc}
\hline Interleukins & Before operation & After operation & $P_{1}$ value & Controls \\
\hline IL-31 & $165.80 \pm 39.03$ & $128.91 \pm 29.48$ & $<0.001$ & $77.24 \pm 25.85$ \\
& $(94.43-240.65)$ & $(53.54-187.54)$ & & $(32.50-142.82)$ \\
IL-33 & $108.85 \pm 7.37$ & $80.93 \pm 9.47$ & 0.005 \\
& $(90.28-123.10)$ & $(58.22-107.63)$ & & $74.29 \pm 22.92$ \\
\hline
\end{tabular}

$P_{1}$ value means the results comparing the interleukin levels in patients with their samples acquiring before and after the operation.

$P_{2}$ value means the results comparing the interleukin levels with the patients' samples acquiring before the operation and those of the controls.

There was no age difference found between patients and controls $(P=0.681)$. A seven-point standard curve was drawn for estimating the concentration of serum IL-31 $\left(R^{2}=\right.$ $0.998)$ and IL-33 $\left(R^{2}=0.999\right)$. The results of serum level of IL31 and IL-33 in EC were shown in Table 2. Both of the serum levels of IL-31 and IL-33 in the patients were dramatically higher than the counterparts of the controls (for IL-31, $P<$ 0.0001 ; for IL-33, $P<0.0001$; Figure 1). The cut-off value of serum IL-31 was about $113.1 \mathrm{pg} / \mathrm{mL}$ (sensitivity: $92.68 \%$, specificity: $94.87 \%$ ) and the area under the ROC curve (AUC) of IL-31 was $0.973 ; P<0.0001 ; 95 \% \mathrm{CI}: 0.945-0.998$. The best cut-off value of serum IL-33 was about $98.42 \mathrm{pg} / \mathrm{mL}$ (sensitivity: 88.64\%, specificity: $97.22 \%$ ); the AUC was 0.929; $P<0.0001$; 95\% CI: 0.86-0.998 (Figure 2).

3.2. Relationship between Serum IL-31/IL-33 and Clinicopathologic Factors. The clinical characteristics include the age,
BMI, tumor stages, depth of invasion, lymphaden metastasis, and distant metastasis. The associative results between serum levels of IL-31 and IL-33 and clinical factors in patients with EC were listed in Table 3. It revealed that serum IL-31 was related to tumor stages $(P=0.024)$ and serum IL-33 was close to the disease process: tumor stages $(P=0.035)$, depth of invasion $(P=0.008)$, and existence of node metastases $(P=0.029)$ and distant metastases $(P=0.036)$. However, to our knowledge, there are no publications on the clinical characteristics of IL-31/IL-33 in EC.

3.3. Serum Levels of Tumor Markers. Furthermore, we analyzed the ROC of the tumor markers in the EC patients. The mean concentration of serum CEA level was $5.22 \pm$ $14.33 \mathrm{ng} / \mathrm{mL}$ (sensitivity: $80 \%$, specificity: $45.71 \%$ ), and the AUC was $0.644 ; P=0.027 ; 95 \%$ CI: $0.524-0.764$. The mean concentration of serum CA-125 level was $25.80 \pm 17.78 \mathrm{U} / \mathrm{mL}$ 


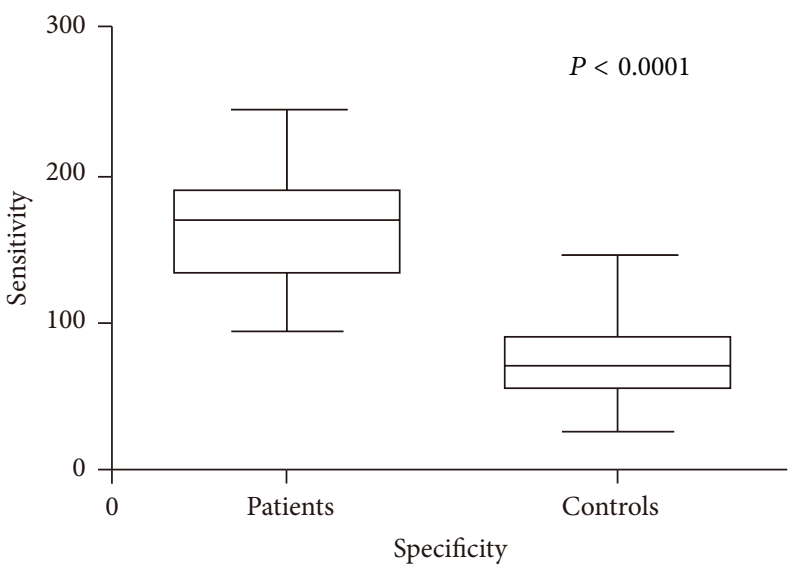

(a)

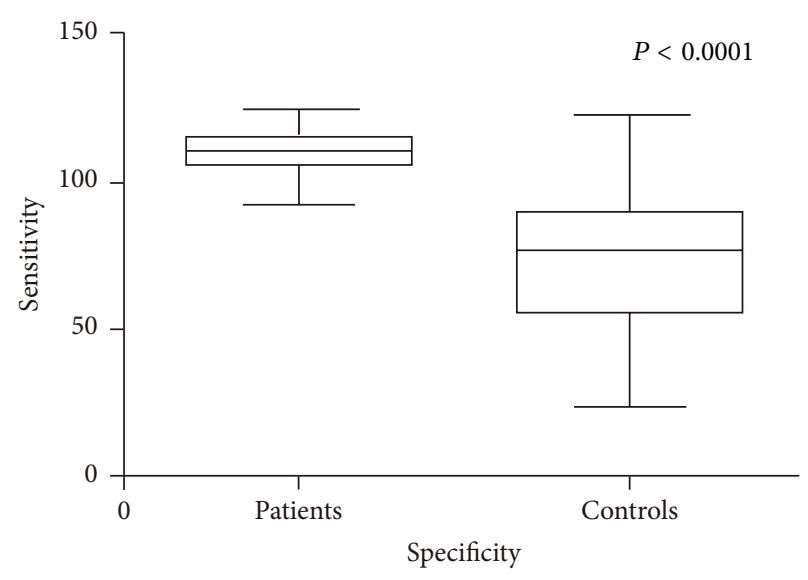

(b)

FIGURE 1: (a) Comparison of the serum levels of IL-31 between the patients and controls. (b) Comparison of the serum levels of IL-33 between the patients and controls.

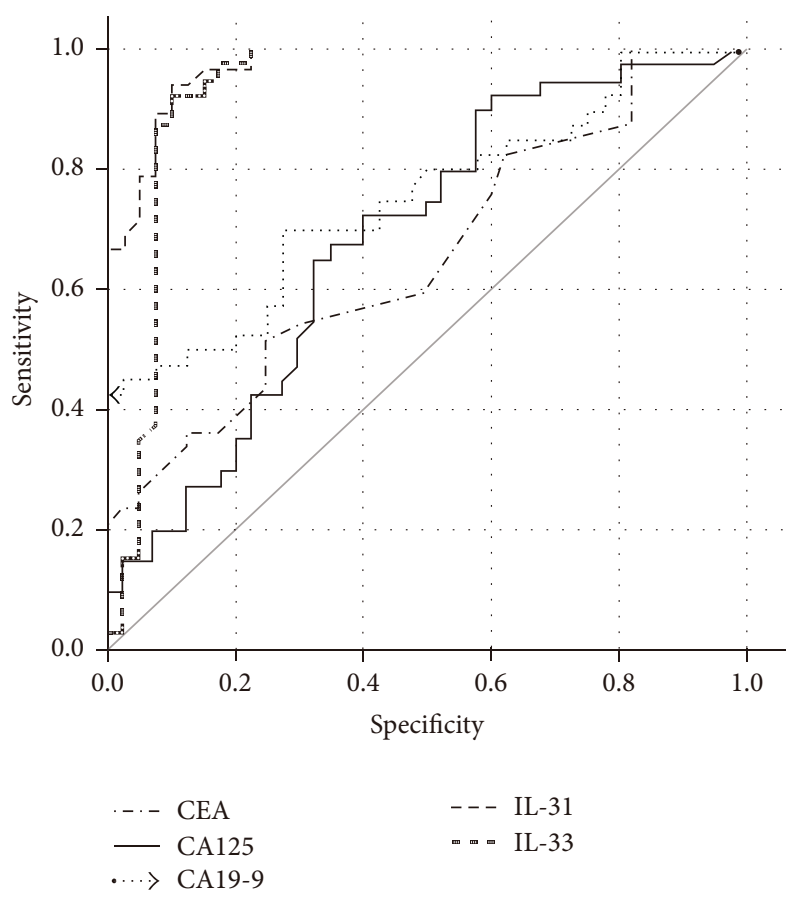

FIGURE 2: Comparison of the ROC curves among the serum levels of CEA, CA-125, CA19-9, IL-31, and IL-33.

(sensitivity: $72.72 \%$, specificity: $46.38 \%$ ), and the AUC was $0.684 ; P=0.005 ; 95 \%$ CI: $0.567-0.801$. The mean concentration of serum CA19-9 level was $60.69 \pm 63.54 \mathrm{U} / \mathrm{mL}$ (sensitivity: $81.33 \%$, specificity: $47.86 \%$ ), and the AUC was 0.751; $P<0.001 ; 95 \%$ CI: 0.645-0.857 (Figure 2). Based on our data, there seemed to be no relationship between the serum levels of the interleukins and the tumor markers (data not shown). Another logistic analysis of multitarget diagnostic value evaluation verified that the sensitivity and specificity combination of IL-31 and IL-33 were also higher than the counterparts of tumor markers (Table 4).

\section{Discussion}

Tumor growth and metastases are regulated by several pathological processes and mediated by inhibitors and stimulators. The close association between inflammation and cancer has been well studied in that the inflammation orchestrates the tumor microenvironment [27, 28]. Previously, researches have established that $\mathrm{T}$ cell-mediated inflammation plays a major role in the development of most cancers.

IL-33 proved signaling via the receptor-related protein of ST2L, which is widely expressed on Th cells, and mast cells and the receptor can be higher in cancer patients than in healthy controls [10, 20, 29]. IL-33 can be produced by necrotic or inflamed tissues and used as an alarmin for damaging inflammation [30]. Sun et al. reported that IL-33 levels in the serum of gastric cancer patients were significantly elevated in comparison with that of healthy volunteers, and higher serum levels of IL-33 in gastric cancer patients were found to correlate with several poor prognostic factors like depth of invasion, distant metastasis, and advanced stage and serum immunoglobulin levels can be elevated by either stimulation of cells with IL-33 or binding of IL-33 to the ST2L in the process of some cancers [25]. To the tumor specific immune response, it can also be turned down or even blinded by some Th2 type cytokines [30]. Therefore, IL-33 can join in Th2 immunity and stimulate the ST2L receptor on various inflammatory cells, playing an important role in the release of proinflammatory factors [31]. Following those factors, vascular changes can be induced, including upgrading the permeability of the microvasculature and vasodilatation and recruiting inflammatory cells [32].

Furthermore, IL-33 is considered closely associated with IL-18 [16], which has been demonstrated novelly to be upregulated in cancer patients [33]; thus IL-33 may also highlight a close association with tumor. Previously, researches had confirmed it in gastric cancer patients [25].

In the cancer group, we found that the serum level of IL33 was higher than in controls $(P<0.0001)$. We also found 
TABLE 3: Correlations of serum IL-31 and IL-33 levels with clinical factors in patients with EC (pg/mL). Data is shown as mean \pm standard deviation (SD).

\begin{tabular}{|c|c|c|c|c|c|}
\hline Characters & Number of patients (\%) & Serum IL-31 & $P_{1}$ value $^{*}$ & Serum IL-33 & $P_{2}$ value $^{* *}$ \\
\hline \multicolumn{6}{|l|}{ Age (years) } \\
\hline$<60$ & $108(67.5 \%)$ & $170.87 \pm 33.05$ & 0.335 & $110.36 \pm 7.69$ & 0.062 \\
\hline$\geq 60$ & $52(32.5 \%)$ & $181.37 \pm 29.11$ & & $105.73 \pm 7.70$ & \\
\hline \multicolumn{6}{|l|}{ BMI } \\
\hline $18.5 \leq \mathrm{BMI} \leq 24.99$ & $72(45 \%)$ & $162.76 \pm 37.98$ & 0.859 & $108.11 \pm 8.36$ & 0.818 \\
\hline $25 \leq \mathrm{BMI} \leq 28$ & $68(42.5 \%)$ & $169.85 \pm 38.89$ & & $109.71 \pm 6.77$ & \\
\hline BMI > 28 & $20(12.5 \%)$ & $163 \pm 50.57$ & & $108.6 \pm 6.6$ & \\
\hline \multicolumn{6}{|l|}{ Tumor stages } \\
\hline $\mathrm{S} 1$ and $\mathrm{S} 2$ & $88(55 \%)$ & $164.18 \pm 22.09$ & 0.024 & $106.66 \pm 6.27$ & 0.035 \\
\hline $\mathrm{S} 3$ and $\mathrm{S} 4$ & $72(45 \%)$ & $186.63 \pm 37.80$ & & $111.54 \pm 7.88$ & \\
\hline \multicolumn{6}{|l|}{ Depth of invasion } \\
\hline $\mathrm{T} 1$ and $\mathrm{T} 2$ & $108(67.5 \%)$ & $169.04 \pm 29.38$ & 0.182 & $106.53 \pm 6.27$ & 0.008 \\
\hline $\mathrm{T} 3$ and $\mathrm{T} 4$ & $52(32.5 \%)$ & $183.01 \pm 34.84$ & & $112.72 \pm 7.62$ & \\
\hline \multicolumn{6}{|l|}{ Lymph node metastasis } \\
\hline Absent & $124(77.5 \%)$ & $169.86 \pm 27.08$ & 0.104 & $107.50 \pm 4.45$ & 0.029 \\
\hline Present & $36(22.5 \%)$ & $189.52 \pm 37.85$ & & $113.52 \pm 9.27$ & \\
\hline \multicolumn{6}{|l|}{ Distant metastasis } \\
\hline M0 & $120(75 \%)$ & $169.50 \pm 29.51$ & 0.100 & $107.24 \pm 6.20$ & 0.014 \\
\hline M1 & $40(25 \%)$ & $188.63 \pm 35.80$ & & $113.71 \pm 8.76$ & \\
\hline
\end{tabular}

${ }^{*} P_{1}$ value means the results of IL-31 with the clinical factors.

${ }^{* *} P_{2}$ value means the results of IL-33 with the clinical factors.

TABLE 4: The value evaluation of multidiagnostic indicators.

\begin{tabular}{lccc}
\hline Indicators & AUC & Sensitivity (\%) & Specificity (\%) \\
\hline IL-31 + IL-33 & 0.931 & $93.86 \%$ & $97.31 \%$ \\
CEA + CA125 & 0.701 & $81.32 \%$ & $51.17 \%$ \\
CEA + CA19-9 & 0.751 & $82.01 \%$ & $60.72 \%$ \\
CA125 + CA19-9 & 0.807 & $85.44 \%$ & $67.99 \%$ \\
CEA + CA125 + CA19-9 & 0.807 & $87.56 \%$ & $76.32 \%$ \\
\hline
\end{tabular}

that the clinical characteristic status correlates with the IL33 values. With a more malignant factor, the serum IL-33 got a higher level and was associated with the tumor stages $(P=0.035)$, depth of invasion $(P=0.008)$, distant metastasis $(P=0.014)$, and lymph node metastasis $(P=0.029)$. The results confirmed our former hypothesis that serum IL33 developed with the process of EC. In particular, IL-33 cut-off of $98.42 \mathrm{pg} / \mathrm{mL}$ yields the best sensitivity of $88.64 \%$ and specificity of $97.22 \%$. In our study, the serum IL-33 is commonly elevated accompanied with some serious clinical factors, like higher tumor stages, depth of invasion, and existence of lymph node metastasis and distant metastasis, which may be further suggested to be related to the progress of the adenocarcinoma.

In tumor patients, IL-31 often produced by damaged and inflamed tissues, especially human mast cell, can also join the T helper type 2 (Th2) responses $[34,35]$. In EC patients of our study, although the serum level of IL-31 was higher than the one in controls, the internal relations with the clinical characteristics were less clear than the counterparts of IL-33. We hypothesized that the IL-31 may be secreted by mast cells after IL-33 stimulation. Comparing with the tumor markers of CEA, CA-125, and CA19-9, the serum IL-31 is still equipped with a better sensitivity (92.68\%) and specificity (94.87\%), using a cut-off value at $113.1 \mathrm{pg} / \mathrm{mL}$.

In literature, preoperational tumor markers' levels of CA125, CEA, and CA19-9 were related to the stage of the disease, myometrial invasion depth, peritoneal cytology, and lymph node metastasis [36-38]. However, the role of those markers in diagnosis of EC is unsatisfied.

CA-125 is a glycoprotein tumor antigen related to epithelial ovarian cancer, which is particularly used for monitoring disease activity for follow-up [39]. Several investigators have presented a sensitivity varying from $24 \%$ to $90 \%$ in diagnosis of EC [38]. However, the up-expression of serum CA-125 and CA19-9 was detected in only $10 \%$ of patients of EC with stages I and II [40]. The following studies illustrated that among the EC patients only $15 \%$ of stage I, 33\% of stage II, and $62 \%$ of stage III have elevated CA-125 levels [41]. CA19-9, a marker related to gastrointestinal cancers, has a sensitivity varying from $11 \%$ to $51.5 \%$ and a poor specificity in EC [38]. CEA is a marker discovered in patients with colon adenocarcinoma, which is increased in $35 \%$ of patients with EC, relating to the size of the uterus and stage of disease [42], and there seems no correlation between lymphoplasmacytic infiltrations of tumor cells and the serum level of CEA in EC [43].

In our research, the sensitivity of CEA was about $80 \%$ and specificity was $45.71 \%$, the sensitivity and specificity of CA125 were about $72.72 \%$ and $46.38 \%$, and the counterparts of 
CA19-9 were $81.33 \%$ and $47.86 \%$, which were confirmed with the literature and lower than the IL-33 and IL-31.

In addition, we were unable to illustrate the correlation between IL-31/IL-33 and endometritis. Those patients with endometritis or pelvic inflammation are commonly admitted to the outpatients department, diagnosed by physical examination, and cured by nonoperation methods; thus we failed to acquire enough cases of peripheral blood and tissues of endometritis to set another control group and there seemed to be no similar studies published before. The further implicit function-promotor of carcinogenesis or reflection of systemic inflammation-remains to be clarified.

Because most patients received operation less than 18 months, the follow-up studies are still undertaken, and the correlation between IL-31/IL-33 and the overall survival time of the endometrial cancer patients is still under research.

\section{Conclusion}

To our knowledge, this is the first clinical research describing a possible relationship between IL-31 and IL-33 and EC. Comparing sensitivity and specificity of some tumor markers, we suppose that elevated serum IL-33 and IL-31 levels, especially IL-33, may relate to the process of EC and could be useful biomarkers for the diagnosis of that disease. However, large prospective clinical studies involving $\mathrm{ICH}$ with endometritis tissues need to be further researched to check the role of the cytokines as new potential tumor markers in EC.

\section{Competing Interests}

None of the authors has any potential competing interests.

\section{Acknowledgments}

This work was supported by the National Natural Science Foundation of China (no. 81572573).

\section{References}

[1] X. Yu, B. Zhou, Z. Zhang et al., "Significant association between IL-32 gene polymorphisms and susceptibility to endometrial cancer in Chinese Han women," Tumor Biology, vol. 36, no. 7, pp. 5265-5272, 2015.

[2] G. M. Lentz, R. A. Lobo, D. M. Gershenson, and V. L. Katz, Comprehensive Gynecology, Elsevier Health Sciences, 2012.

[3] N. Colombo, E. Preti, F. Landoni et al., "Endometrial cancer: ESMO clinical practice guidelines for diagnosis, treatment and follow-up," Annals of Oncology, vol. 22, supplement 6, pp. vi35vi39, 2011.

[4] X. Li, S. Zheng, S. Chen, F. Qin, S. Lau, and Q. Chen, "Trends in gynaecological cancers in the largest obstetrics and gynaecology hospital in China from 2003 to 2013," Tumor Biology, vol. 36, no. 7, pp. 4961-4966, 2015.

[5] F. Amant, P. Moerman, P. Neven, D. Timmerman, E. Van Limbergen, and I. Vergote, "Endometrial cancer," The Lancet, vol. 366, no. 9484, pp. 491-505, 2005.
[6] S. Frantz, K. A. Vincent, O. Feron, and R. A. Kelly, "Innate immunity and angiogenesis," Circulation Research, vol. 96, no. 1, pp. 15-26, 2005.

[7] S. I. Grivennikov and M. Karin, "Inflammation and oncogenesis: a vicious connection," Current Opinion in Genetics and Development, vol. 20, no. 1, pp. 65-71, 2010.

[8] I. H. Ozbudak, S. Karaveli, T. Simsek, G. Erdogan, and E. Pestereli, "Neoangiogenesis and expression of hypoxia-inducible factor $1 \alpha$, vascular endothelial growth factor, and glucose transporter-1 in endometrioid type endometrium adenocarcinomas," Gynecologic Oncology, vol. 108, pp. 603-608, 2008.

[9] N. Ferrara, "Role of vascular endothelial growth factor in the regulation of angiogenesis," Kidney International, vol. 56, no. 3, pp. 794-814, 1999.

[10] R. Smith-Bindman, E. Weiss, and V. Feldstein, "How thick is too thick? When endometrial thickness should prompt biopsy in postmenopausal women without vaginal bleeding," Ultrasound in Obstetrics \& Gynecology, vol. 24, no. 5, pp. 558-565, 2004.

[11] R. Angioli, F. Plotti, S. Capriglione et al., "The role of novel biomarker HE4 in endometrial cancer: a case control prospective study," Tumor Biology, vol. 34, no. 1, pp. 571-576, 2013.

[12] S. Pflanz, L. Hibbert, J. Mattson et al., "WSX-1 and glycoprotein 130 constitute a signal-transducing receptor for IL-27," The Journal of Immunology, vol. 172, no. 4, pp. 2225-2231, 2004.

[13] M. L. Castellani, V. Salini, S. Frydas et al., "Interleukin-31: a new cytokine involved in inflammation of the skin," International Journal of Immunopathology and Pharmacology, vol. 19, no. 1, pp. 1-4, 2006.

[14] W. K. Ip, C. K. Wong, M. L. Y. Li, P. W. Li, P. F. Y. Cheung, and C. W. K. Lam, "Interleukin-31 induces cytokine and chemokine production from human bronchial epithelial cells through activation of mitogen-activated protein kinase signalling pathways: implications for the allergic response," Immunology, vol. 122, no. 4, pp. 532-541, 2007.

[15] V. Carriere, L. Roussel, N. Ortega et al., "IL-33, the IL-1-like cytokine ligand for ST2 receptor, is a chromatin-associated nuclear factor in vivo," Proceedings of the National Academy of Sciences of the United States of America, vol. 104, no. 1, pp. 282287, 2007.

[16] C. A. Dinarello, "Interleukin-1, interleukin-1 receptors and interleukin-1 receptor antagonist," International Reviews of Immunology, vol. 16, no. 5-6, pp. 457-499, 1998.

[17] H. Park, D. Byun, T. S. Kim et al., "Enhanced IL-18 expression in common skin tumors," Immunology Letters, vol. 79, no. 3, pp. 215-219, 2001.

[18] S. A. L. Eissa, S. A. Zaki, S. M. El-Maghraby, and D. Y. Kadry, "Importance of serum IL-18 and RANTES as markers for breast carcinoma progression," Journal of the Egyptian National Cancer Institute, vol. 17, no. 1, pp. 51-55, 2005.

[19] R. A. Merendino, S. Gangemi, A. Ruello et al., "Serum levels of interleukin-18 and sICAM-1 in patients affected by breast cancer: preliminary considerations," The International Journal of Biological Markers, vol. 16, no. 2, pp. 126-129, 2001.

[20] J. Schmitz, A. Owyang, E. Oldham et al., "IL-33, an interleukin1-like cytokine that signals via the IL-1 receptor-related protein ST2 and induces T helper type 2-associated cytokines," Immunity, vol. 23, no. 5, pp. 479-490, 2005.

[21] A. M. Küchler, J. Pollheimer, J. Balogh et al., "Nuclear interleukin-33 is generally expressed in resting endothelium but rapidly lost upon angiogenic or proinflammatory activation," The American Journal of Pathology, vol. 173, no. 4, pp. 1229-1242, 2008 . 
[22] Y.-S. Choi, H.-J. Choi, J.-K. Min et al., "Interleukin-33 induces angiogenesis and vascular permeability through ST2/TRAF6mediated endothelial nitric oxide production," Blood, vol. 114, no. 14, pp. 3117-3126, 2009.

[23] W. Naumnik, B. Naumnik, K. Niewiarowska, M. Ossolinska, and E. Chyczewska, "Novel cytokines: IL-27, IL-29, IL-31 and IL33. Can they be useful in clinical practice at the time diagnosis of lung cancer?” Experimental Oncology, vol. 34, no. 4, pp. 348353, 2012.

[24] S.-F. Chen, S. Nieh, S.-W. Jao et al., "The paracrine effect of cancer-associated fibroblast-induced interleukin-33 regulates the invasiveness of head and neck squamous cell carcinoma," The Journal of Pathology, vol. 231, no. 2, pp. 180-189, 2013.

[25] P. Sun, Q. Ben, S. Tu, W. Dong, X. Qi, and Y. Wu, "Serum interleukin-33 levels in patients with gastric cancer," Digestive Diseases and Sciences, vol. 56, no. 12, pp. 3596-3601, 2011.

[26] S. Pecorelli, "Revised FIGO staging for carcinoma of the vulva, cervix, and endometrium," International Journal of Gynecology and Obstetrics, vol. 105, no. 2, pp. 103-104, 2009.

[27] F. Balkwill and A. Mantovani, "Inflammation and cancer: back to Virchow?” The Lancet, vol. 357, no. 9255, pp. 539-545, 2001.

[28] W. Zou and N. P. Restifo, "TH17 cells in tumour immunity and immunotherapy," Nature Reviews Immunology, vol.10, no. 4, pp. 248-256, 2010.

[29] D. Bergis, V. Kassis, A. Ranglack et al., "High serum levels of the interleukin-33 receptor soluble ST2 as a negative prognostic factor in hepatocellular carcinoma," Translational Oncology, vol. 6, no. 3, pp. 311-318, 2013.

[30] A. Sharma, M. Rajappa, A. Saxena, and M. Sharma, "Cytokine profile in Indian women with cervical intraepithelial neoplasia and cancer cervix," International Journal of Gynecological Cancer, vol. 17, no. 4, pp. 879-885, 2007.

[31] F. Cevikbas and M. Steinhoff, "IL-33: a novel danger signal system in atopic dermatitis," Journal of Investigative Dermatology, vol. 132, no. 5, pp. 1326-1329, 2012.

[32] M. Enoksson, K. Lyberg, C. Möller-Westerberg, P. G. Fallon, G. Nilsson, and C. Lunderius-Andersson, "Mast cells as sensors of cell injury through IL-33 recognition," The Journal of Immunology, vol. 186, no. 4, pp. 2523-2528, 2011.

[33] M. R. Haghshenas, S. V. Hosseini, M. Mahmoudi, M. SaberiFirozi, S. Farjadian, and A. Ghaderi, "IL-18 serum level and IL-18 promoter gene polymorphism in Iranian patients with gastrointestinal cancers," Journal of Gastroenterology and Hepatology, vol. 24, no. 6, pp. 1119-1122, 2009.

[34] P. N. Pushparaj, H. K. Tay, S. C. H'ng et al., "The cytokine interleukin-33 mediates anaphylactic shock," Proceedings of the National Academy of Sciences of the United States of America, vol. 106, no. 24, pp. 9773-9778, 2009.

[35] C. Cornelissen, J. Lüscher-Firzlaff, J. M. Baron, and B. Lüscher, "Signaling by IL-31 and functional consequences," European Journal of Cell Biology, vol. 91, no. 6-7, pp. 552-566, 2012.

[36] C.-H. Hsieh, C.-C. ChangChien, H. Lin et al., "Can a preoperative CA 125 level be a criterion for full pelvic lymphadenectomy in surgical staging of endometrial cancer?" Gynecologic Oncology, vol. 86, no. 1, pp. 28-33, 2002.

[37] G. Scambia, A. Gadducci, P. B. Panici et al., "Combined use of CA 125 and CA 15-3 in patients with endometrial carcinoma," Gynecologic Oncology, vol. 54, no. 3, pp. 292-297, 1994.

[38] P. L. Cherchi, S. Dessole, G. A. Ruiu et al., "The value of serum CA 125 and association CA 125/CA 19-9 in endometrial carcinoma," The European Journal of Gynaecological Oncology, vol. 20, no. 4, pp. 315-317, 1999.
[39] J. Nicklin, M. Janda, V. Gebski et al., "The utility of serum CA125 in predicting extra-uterine disease in apparent early-stage endometrial cancer," International Journal of Cancer, vol. 131, no. 4, pp. 885-890, 2012.

[40] N. Takeshima, Y. Shimizu, S. Umezawa et al., "Combined assay of serum levels of CA125 and CA19-9 in endometrial carcinoma," Gynecologic Oncology, vol. 54, no. 3, pp. 321-326, 1994.

[41] E. P. Beck, M. Wagner, L. Anselmino, F.-J. Xu, R. C. Bast Jr., and W. Jaeger, "Is OVX1 a suitable marker for endometrial cancer?" Gynecologic Oncology, vol. 65, no. 2, pp. 291-296, 1997.

[42] J. R. Van Nagell Jr., E. S. Donaldson, E. G. Wood, and D. M. Goldenberg, "The clinical significance of carcinoembryonic antigen in the plasma and tumors of patients with gynecologic malignancies," Cancer, vol. 42, no. 3, pp. 1527-1532, 1978.

[43] J. R. Van Nagell, W. R. Meeker, J. C. Parker, and J. D. Harralson, "Carcinoembryonic antigen in patients with gynecologic malignancy," Cancer, vol. 35, no. 5, pp. 1372-1376, 1975. 


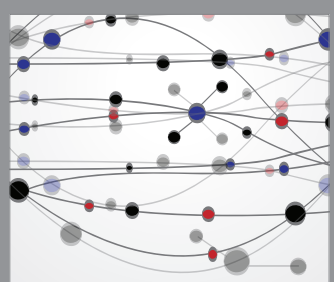

The Scientific World Journal
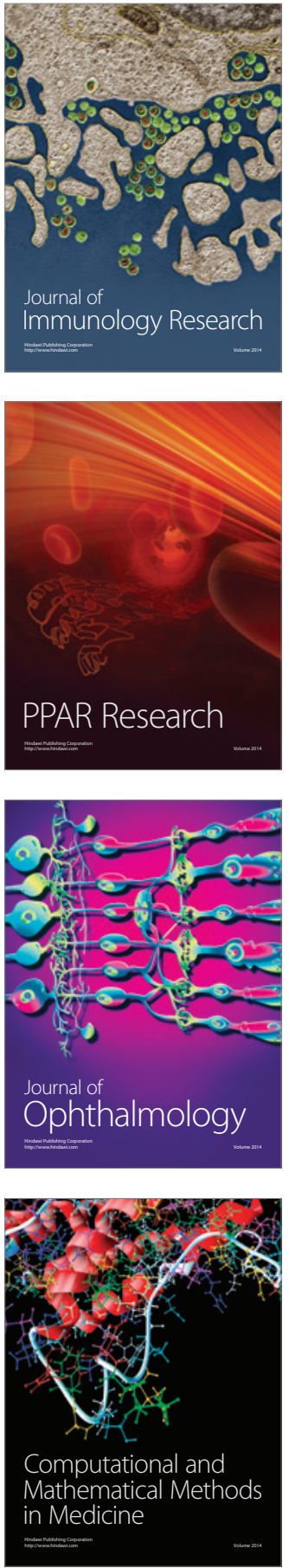

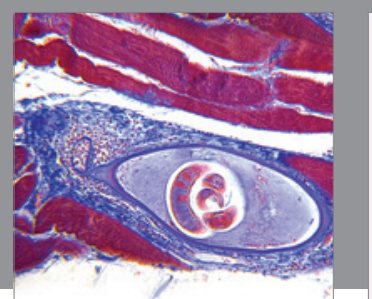

Gastroenterology Research and Practice

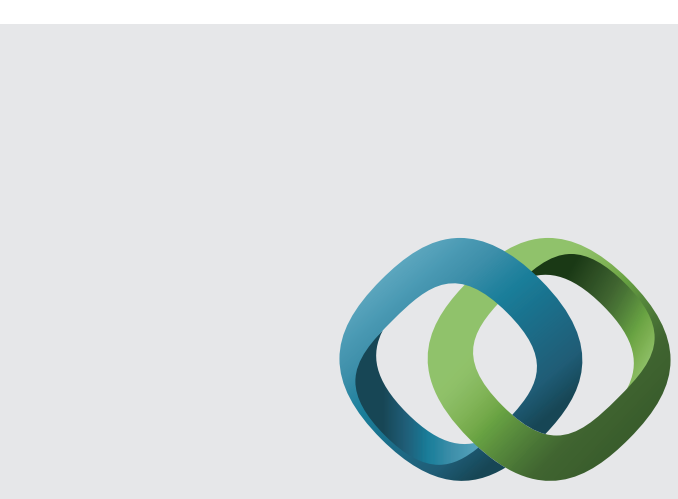

\section{Hindawi}

Submit your manuscripts at

http://www.hindawi.com
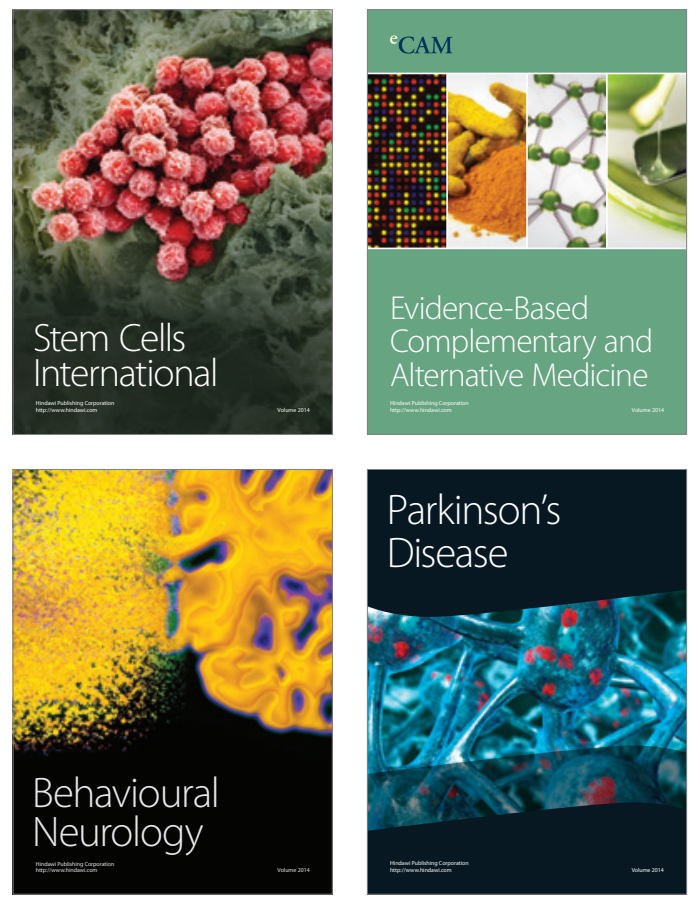
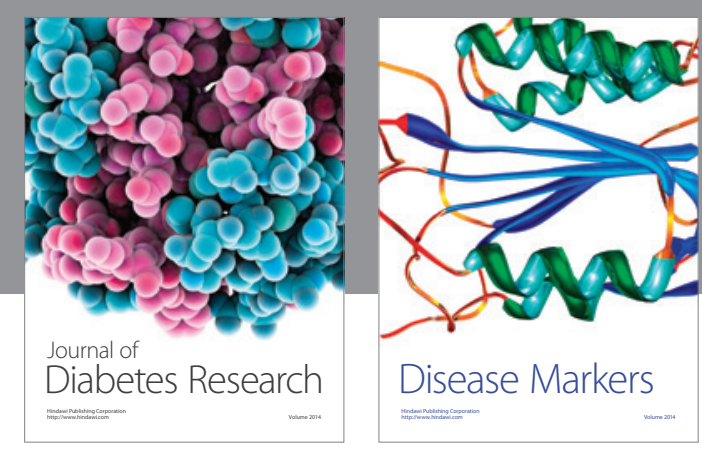

Disease Markers
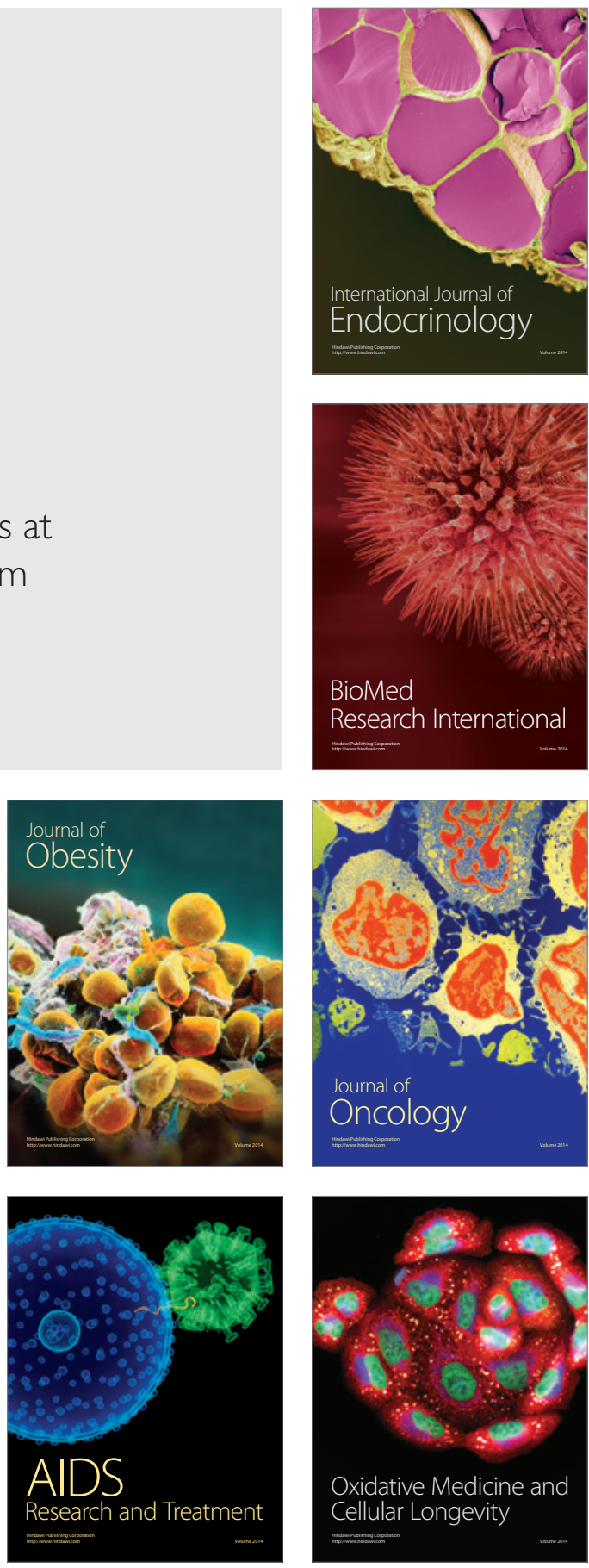